\title{
Alveolar blood clots and platelet-rich fibrin induce in vitro fibroblast proliferation and migration
}

\author{
MIHAI BUCUR ${ }^{1,2 *}$, CAROLINA CONSTANTIN ${ }^{3,4 *}$, MONICA NEAGU $^{3-5 *}$, SABINA ZURAC $^{1,3^{*}}$, \\ OCTAVIAN DINCA ${ }^{1,2}$, CRISTIAN VLADAN ${ }^{1,2}$, MIRELA CIOPLEA $^{3}$, CRISTIANA POPP ${ }^{3}$, \\ LUCIANA NICHITA $^{1,3}$ and ECATERINA IONESCU ${ }^{1,6}$
}

\author{
${ }^{1}$ Faculty of Dental Medicine, 'Carol Davila' University of Medicine and Pharmacy, 020021 Bucharest; \\ ${ }^{2}$ Department of Oro-Maxillofacial Surgery, 'Prof. Dr. Dan Theodorescu' Clinical Hospital of \\ Oro-Maxillofacial Surgery, 101022 Bucharest; ${ }^{3}$ Department of Pathology, Colentina University Hospital, \\ 020125 Bucharest; ${ }^{4}$ Department of Immunology, 'Victor Babeş' National Institute of Pathology, \\ 050096 Bucharest; ${ }^{5}$ Faculty of Biology, University of Bucharest, 050095 Bucharest; ${ }^{6}$ Ambulatory of Orthodontics, \\ 'Prof. Dr. Dan Theodorescu' Clinical Hospital of Oro-Maxillofacial Surgery, 010221 Bucharest, Romania
}

Received August 17,2018; Accepted September 26, 2018

DOI: $10.3892 / \mathrm{etm} .2018 .7063$

\begin{abstract}
Wound healing process comprises a complex network of cells and molecules that are regulated in order to pursue tissue regeneration. Our study focused on the capacity of alveolar blood clots (ABCs), platelet-rich fibrin (PRF) and plasma rich in growth factors (PRGF) to induce in vitro fibroblasts proliferation and migration as a measure of alveolar regeneration. Using cellular impedance with xCELLigence technology we quantified the proliferation and the migration capacity of L929 fibroblast standard cell line in the presence of 4 different $\mathrm{ABCs}$ and 3 different PRFs harvested from healthy individuals during standard tooth extraction. We obtained a clear cellular proliferation induced by the compounds mainly after $24 \mathrm{~h}$ of cultivation, in a dose-dependent manner. After
\end{abstract}

Correspondence to: Dr Monica Neagu, Department of Pathology, Colentina University Hospital, 19-21 Stefan cel Mare Blv., 020125 Bucharest, Romania

E-mail: neagu.monica@gmail.com

*Contributed equally

Abbreviations: PRF, platelet-rich fibrin; PRGFs, plasma rich in growth factors; GF, fibroblasts from human gingiva; SFs, skin fibroblasts; MMP, matrix metalloproteinase; ECM, extracellular matrix; PDGF, platelet-derived growth factor; EGF, epidermal growth factor; VEGF, vascular endothelial growth factor; SDF-1, stromal cell-derived factor-1; IGF-1, insulin-like growth factor-1; bFGF, fibroblast growth factor basic; TGF- $\beta$, transforming growth factor- $\beta$; HGF, hepatocyte growth factor; IL, interleukin; MCP-1, monocyte chemoattractant protein-1

Key words: fibroblasts, cellular impedance, alveolar blood clot, platelet-rich fibrin, plasma rich in growth factors, tissue regeneration, wound healing
$48 \mathrm{~h}$ of cultivation we registered activated proliferation, but slightly decreased compared to the $24 \mathrm{~h}$ profile. Our data confirm that the presence of the blood clot is involved in the regenerative processes. The migratory capacity of fibroblasts was statistically activated by the PL compounds while not affected by the tested PRFs. The chemical mediators present within the blood clot, either produced by inflammatory cells captive within, or by endothelial or mesenchymal cells induced fibroblastic proliferation and subsequent collagen deposition.

\section{Introduction}

Fibroblasts are the primary source of most extracellular matrix components, are abundant in skin, participating in the turnover of extracellular matrix as one of the main events in wound healing (1). The density of the fibroblasts varies in different areas of skin as well as the collagen and elastin quantity varies within anatomic location (2). The role of fibroblasts is complex; they intervene in fibroplasias and angiogenesis (3) but also in inflammation, granulation tissue formation and scar remodeling $(4,5)$. Their involvement in regeneration was revisited by many recent studies related to scar-free wound healing process in oral mucosa, an area distinct from regular skin with respect to fibroblasts phenotype (6-8). The mechanisms of scar formation following skin damage are still to be elucidated although several reports indicate that wound healing in oral mucosa is faster and trigger a reduced inflammation compared with regular skin fibroblasts. These differences were attributed to a specific phenotype of fibroblasts from human gingiva (GF) which is different from other skin fibroblasts (SF). For instance, GF express high levels of molecules involved in regulation of inflammation and MMPs favorable for rapid resolution of inflammation and ECM remodeling, which is typical for scarfree wound healing, while SF exhibit a profibrotic, scar-prone phenotype (9). Moreover, recent studies claim involvement of some cell surface specific molecules in sustaining the profi- 
brotic phenotype. Thus, studies in mice have linked a high expression of CD26 to a profibrotic phenotype of fibroblasts also associated with an increased level of transforming growth factor- $\beta$ signaling. Therefore, it is possible that CD26-positive fibroblast population that is abundant in human skin but not in gingiva, may differently drive the profibrotic response, scar formation and wound healing (10). A complex meta-analysis regarding the efficacy of platelet concentrates in pulpotomy of human teeth was recently published (11). The enrolled were in the range of 4-25 years and autologous platelet-rich fibrin (PRF) allogeneic lyophilized freeze-dried platelet was used as pulpotomy material compared to calcium hydroxide and mineral trioxide aggregate. Two of the three investigated studies have shown $100 \%$ success of pulpotomy with PRF and the rest of the studies more than $80 \%$. Interestingly when assessing the control groups the differences in regeneration were not statistically significant. This meta-analysis pointed out the need for further studies of the mechanisms that reside in this regeneration (11). Thus, in the light of gathered information we are focusing on an in vitro experimental model to test the proliferation and migration capacity of standard fibroblasts in the presence of normal alveolar blood clots (ABCs), platelets or platelets lysates as the main source of pro-regeneration molecules.

\section{Materials and methods}

$A B C s$ were harvested from normal subjects who underwent elective dental extractions. In each case, a full thickness flap was performed in the area by giving an intra sulcular incision. Hemostasis was obtained and postsurgical instructions were given to the patients. Instructions included applying pressure for $2 \mathrm{~h}$ and a warning not to manipulate the surgical site or attempt to retract the lip to visualize the surgical area, and $20 \mathrm{~min}$ later, the periodontal flap was reflected to visualize alveolar sockets; blood clots were noted in relation to the surgical site and were removed with a curette, in sterile conditions. The site was irrigated with saline solution and flaps were secured back to its original position with 3-0 silk suture.

This study was approved by the Ethics Committee of Colentina University Hospital (no. 63/31.10.2016; Bucharest, Romania), and all patients gave their informed written consent for this study complying with the Declaration of Helsinki.

Four different clots were tested after re-suspending them in $1 \mathrm{ml}$ of cell culture medium without any additional supplements. To remove all cells and cellular debris all the resuspended clots were filtered through $0.22-\mu \mathrm{m}$ sterile filters (EMD Millipore, Billerica, MA, USA). Afterwards, serial dilutions were made in plain cell culture medium as follows: 1:4, 1:8, 1:16, 1:48. The PRF scaffolds were prepared according to the following protocol: ten healthy volunteers in an age range between 18 to 60 years participated in this study. For each individual, 4 tubes of peripheral blood were collected and immediately placed in a pre-programmed centrifuge. Centrifugation was performed according to the following protocol: advanced PRF, sterile plain glass-based vacuum tubes (A-PRF10 tube) (10 ml; centrifuged at $200 \times \mathrm{g}$ for $14 \mathrm{~min}$ ). To produce PRGF we harvested blood from other 10 healthy donors into 9-ml collection tubes containing $0.9 \mathrm{ml}$ of $3.8 \%(\mathrm{wt} / \mathrm{v})$ trisodium citrate. Blood samples were centrifuged at $580 \mathrm{xg}$ for $8 \mathrm{~min}$ at

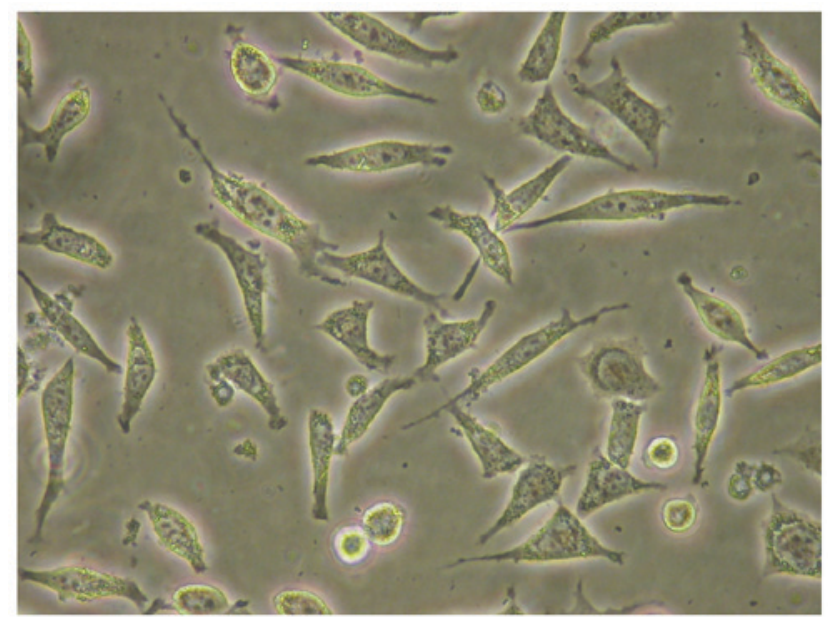

Figure 1. L929 cell line maintained in culture; active proliferation after $24 \mathrm{~h}$ post-seeding; x20 magnification in inverse microscopy.

room temperature in the PRGF-Endoret System IV centrifuge (BTI Biotechnology Institute, S.L., Álava, Spain). Afterward, the whole plasma column over the buffy coat was collected using Endoret kit (BTI Biotechnology Institute, S.L.) avoiding the layer containing leukocytes.

L929 standard skin fibroblast cell line. L929 standard skin fibroblast cell line was purchased from European Collection of Authenticated Cell Cultures (ECACC) and kept in 'Victor Babes' National Institute of Pathology Biobank (Bucharest, Romania). L929 (ECACC; cat. no. 85011425) was maintained in culture according the supplier specification. Briefly, seeding was done at 10,000 cells $/ \mathrm{cm}^{3}$ in DMEM cell culture medium supplemented with $2 \mathrm{mM}$ glutamine and $10 \%$ fetal bovine serum (FBS); cultures were maintained in $5 \% \mathrm{CO}_{2}$ at $37^{\circ} \mathrm{C}$. Cells displayed specific spindle shape morphology (Fig. 1). When reaching $80 \%$ confluence, adherent cells were detached with $0.25 \%$ trypsin/EDTA, cell suspension was counted in an automated Cell Counter Countess (Thermo Fisher Scientific, Inc., Waltham, MA, USA) using Trypan blue exclusion test. Cell culture viability was $100 \%$ and their doubling time for L929 fibroblasts was $14 \mathrm{~h}$. When cell cultures reached $80 \%$ confluence we seeded the fibroblasts in E16 plates at 2,500 cells/well. After $2 \mathrm{~h}$ for controls plain cell culture medium was added (control 1), cell culture medium supplemented with $10 \%$ horse serum (control 2). For testing the ABCs the tested clots were added in serial dilution without any additional serum in the mentioned dilutions. Triplicates of each experimental system were registered. For migration experiments CIM plates were treated with fibronectin $1 \mu \mathrm{g} / \mathrm{ml}$ in sterile PBS (75 $\mu \mathrm{l} /$ well overnight incubation at $4^{\circ} \mathrm{C}$ ) to facilitate cell migration. After incubation fibronectin was totally removed and plates gently washed with sterile PBS twice. Fibroblasts cultivated in medium without horse serum were seeded in the upper chamber at $1 \times 10^{5} / \mathrm{ml}$; in the lower chamber B the compounds were added in triplicates along with controls horse serum $10 \%$ and plain medium.

Real-time monitoring of cell response using impedance technology. We have used this technology to provide the 


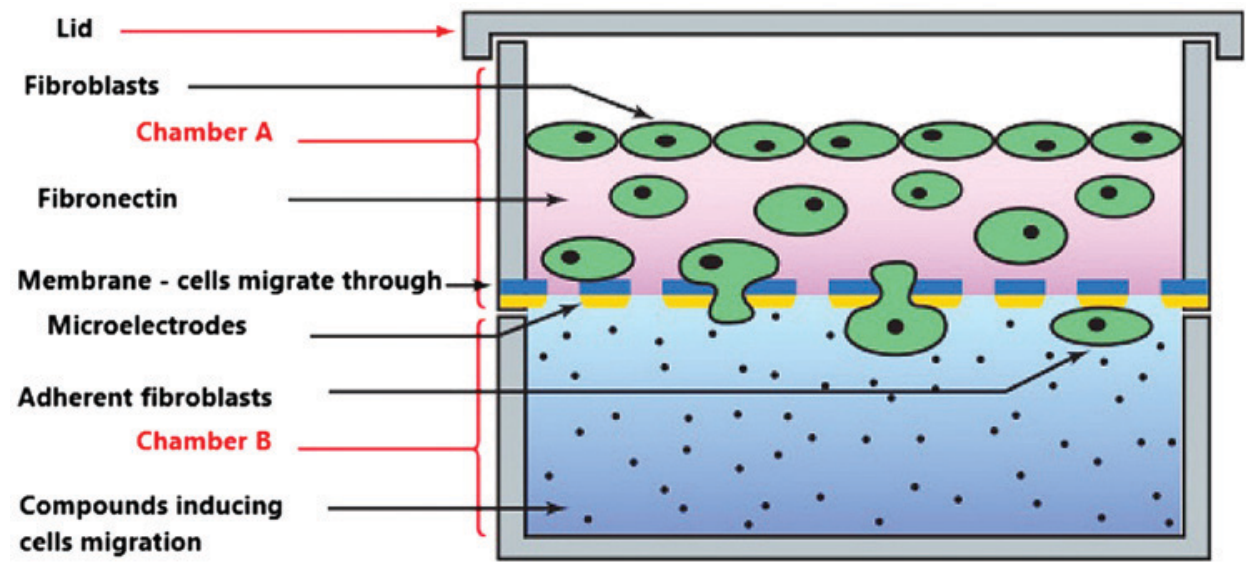

Figure 2. Cell migration takes place from the upper chamber (chamber A) through a membrane that allows only migratory phenotype cells to transverse the membrane and adhere to the impedance electrodes on the lower part of the membrane. Cells migrate towards the lower chamber (chamber B) where the chemoattractants reside. The adherence capacity is quantified as cellular index registered by the microelectrodes similar to the proliferation assay.

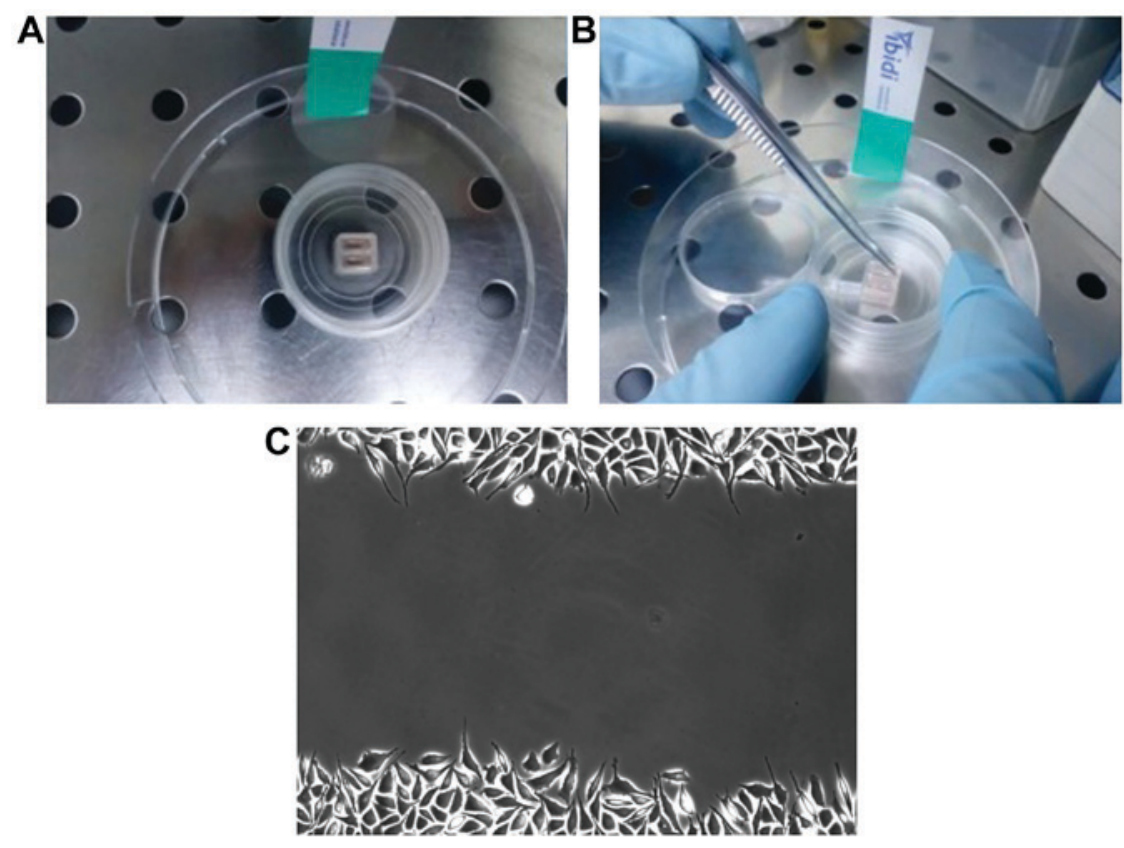

Figure 3. (A) Fibroblasts were seeded at a density of $0.33 \times 10^{6}$ cells/IBIDI plate in a final volume of $1.7 \mathrm{ml}$. (B) After $24 \mathrm{~h}$ of cultivation the silicon frame is removed and (C) in the plate the remaining gap measures $500 \mu \mathrm{m}$.

quantification of cell proliferation as previously reported by us $(12,13)$. Briefly, experiments were performed on non-coated E-16 plates, compatible with RCTA-DP system (both from Roche Applied Science, Penzberg, Upper Bavaria, Germany). Cells were left to adhere for $2 \mathrm{~h}$ in the RTCA DP device at $37^{\circ} \mathrm{C}$ and $5 \% \mathrm{CO}_{2}$. Readings were collected at 1 min intervals for $72 \mathrm{~h}$ and the results reported as normalized cellular index $(\mathrm{CI})$ to the time before addition of the compounds. The assay system expresses cell's impedance in arbitrary CI units; results are presented as overall CI for each sample and time-dynamic evaluation of CI. The same technology was used to investigate the migratory capacity of the tested fibroblasts in the presence of compounds. For the overall CI the software delivers automatically at pre-established time interval the values, in our case at 2, 24 and $48 \mathrm{~h}$. For CI time-dynamic evaluation graphs are also automatically displayed and we have presented the general patterns of cell's evolution. Migration was quantified using CIM-Plate 16 (Roche Applied Science), compatible with RCTA-DP system. The plates are actually a Boyden chamber with an upper and a lower chamber as presented in Fig. 2. Polyethylene terephatalate (PET) membrane has specific pores that accomodate only elongated migratory cells. The lower part of the membrane accomodates the microelectrodes for cellular impedance measurement.

Scratch test. Scratch test for registering the capacity of PRGF and PRF compounds to induce wound healing was used the test for monitoring the actual fibroblasts migration on a standard gap (14). Fibroblasts were seeded at a density of $0.33 \times 10^{6}$ cells/IBIDI plate and $24 \mathrm{~h}$ of cultivation at $37^{\circ} \mathrm{C}$ in $5 \% \mathrm{CO}_{2}$, the silicon frame was removed displaying a standard gap of $500 \mu \mathrm{m}$ (Fig. 3). The plates were washed thoroughly 


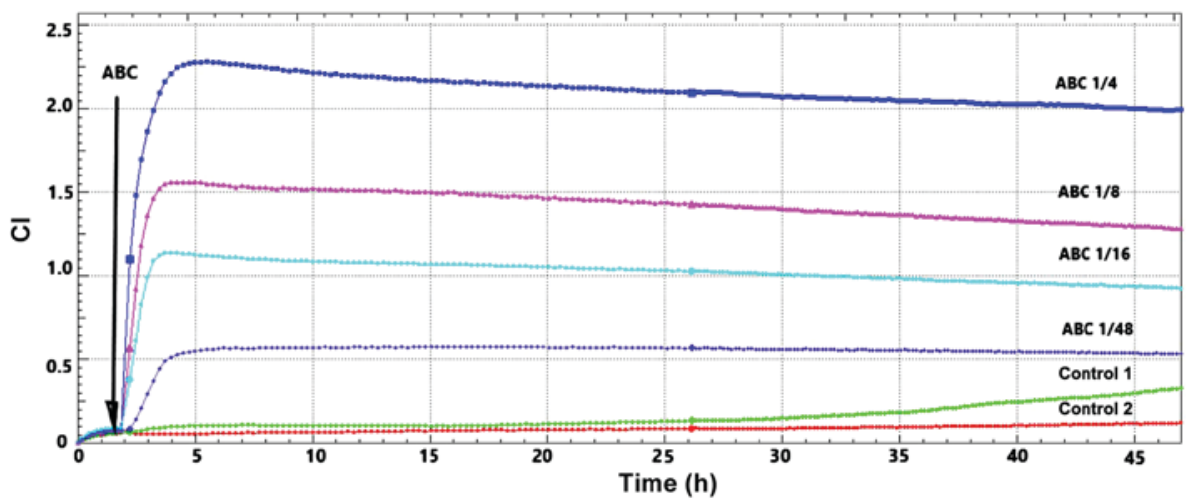

Figure 4. L929 fibroblast proliferation registered for $47 \mathrm{~h}$ of cultivation in $\mathrm{ABC}$ in various serial dilution compared to controls cultivated in plain culture medium (control 1) and culture medium with serum supplements (control 2). Black arrow depicts the moment ABCs in the presented dilutions are introduced in the experimental system (example of an individual experiment CI - mean of triplicates). ABC, alveolar blood clot.
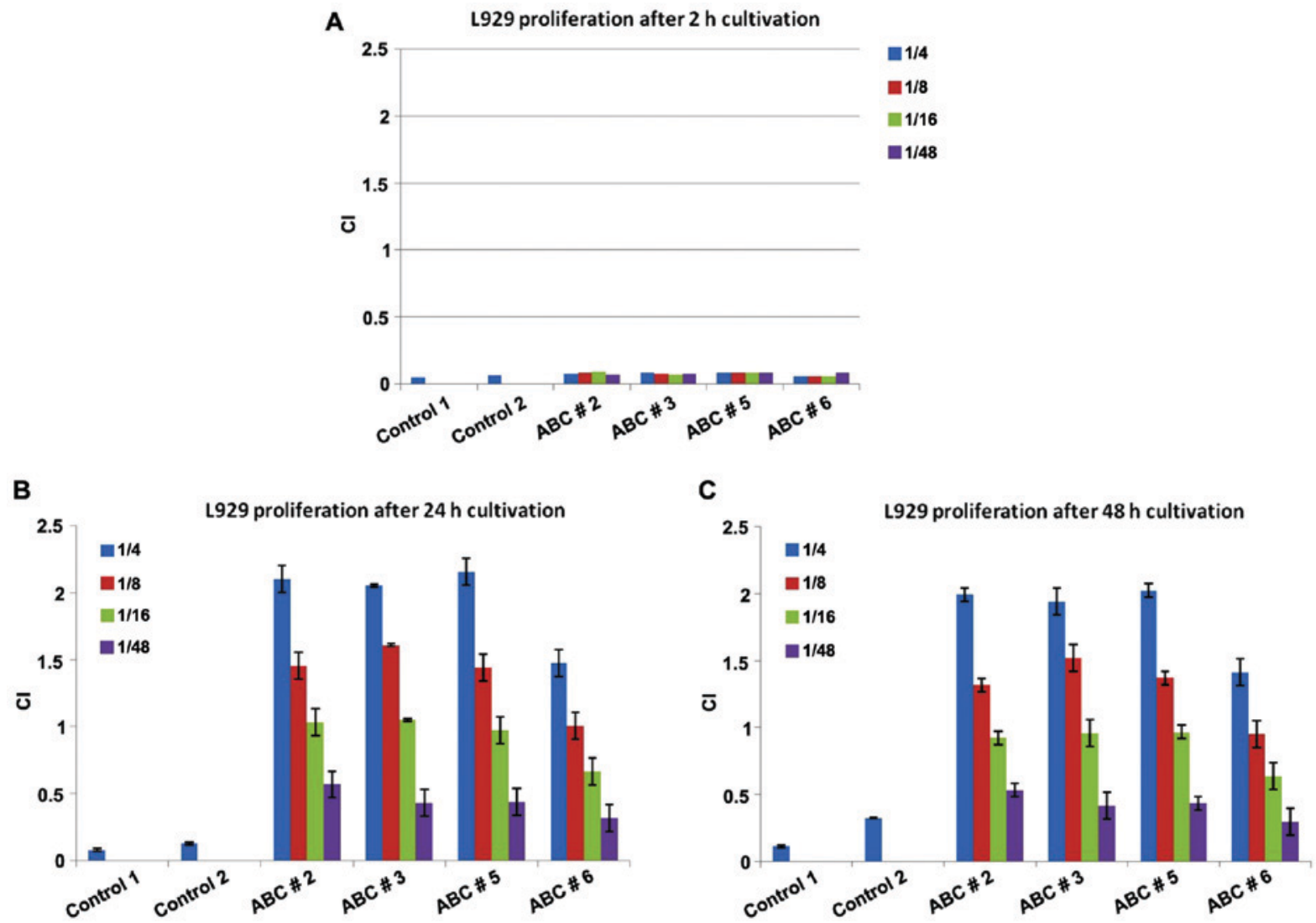

Figure 5. Cellular index (CI) calculated for cultivated fibroblasts in individual $\mathrm{ABC}$ (nos. 2, 3, 5, 6) compared to plain cell culture medium (control 1), cell culture medium supplemented with $10 \%$ horse serum (control 2). A, $2 \mathrm{~h} ; \mathrm{B}, 24 \mathrm{~h} ; \mathrm{C}, 48 \mathrm{~h}$ of cultivation (CI of mean \pm SD for 3 different experiments). p<0.001 when comparing controls with all the corresponding compounds. $\mathrm{ABC}$, alveolar blood clot.

with PBS to remove any debris. Fibroblasts in the presence of $3 \mathrm{ml}$ complete medium with/without $10 \%$ serum (controls) or in the presence of the tested compounds were incubated and migration registered by video capture in BioStation (Nikon Corporation, Tokyo, Japan).

Statistical analysis. All the experiments were performed three times and each system comprised at least triplicates as stated. CI was automatically registered by the software (RTCA 2.1.0 Software; ACEA Biosciences Inc., San Diego, CA, USA) and mean $\pm \mathrm{SD}$ is represented in the figures. For individual repre- sentation of the experiments the mean value of the triplicates was presented as directly registered by the equipment.

\section{Results}

Fibroblasts proliferation. After the first $2 \mathrm{~h}$ of $\mathrm{L} 929$ cell cultivation in E16 plates we introduced serial dilution of different $\mathrm{ABCs}$ along with the appropriate controls. Registering the $\mathrm{CI}$ for $47 \mathrm{~h}$ we noted the same pattern for all the tested compounds (Fig. 4). It can be observed that the proliferation capacity is dose-dependent and perfectly matches the tested 


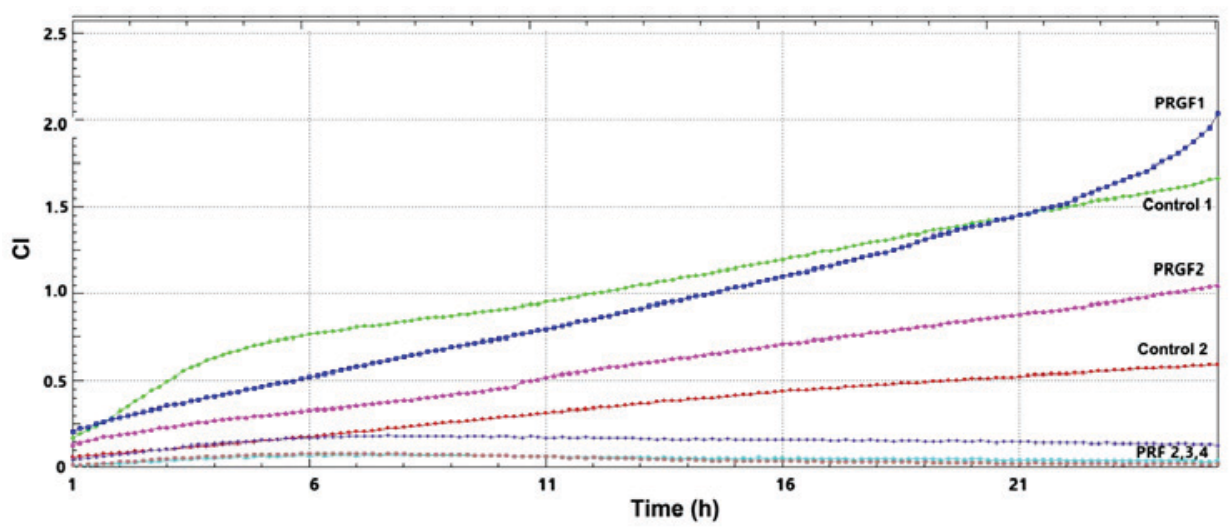

Figure 6. Fibroblast migration registered for $24 \mathrm{~h}$ in the presence of PRGF1 and 2, PRF2-4 compared to control 1 (fibroblasts migrating toward complete culture medium) and control 2 (fibroblasts migrating toward plain medium). Representation of CI mean for triplicates.

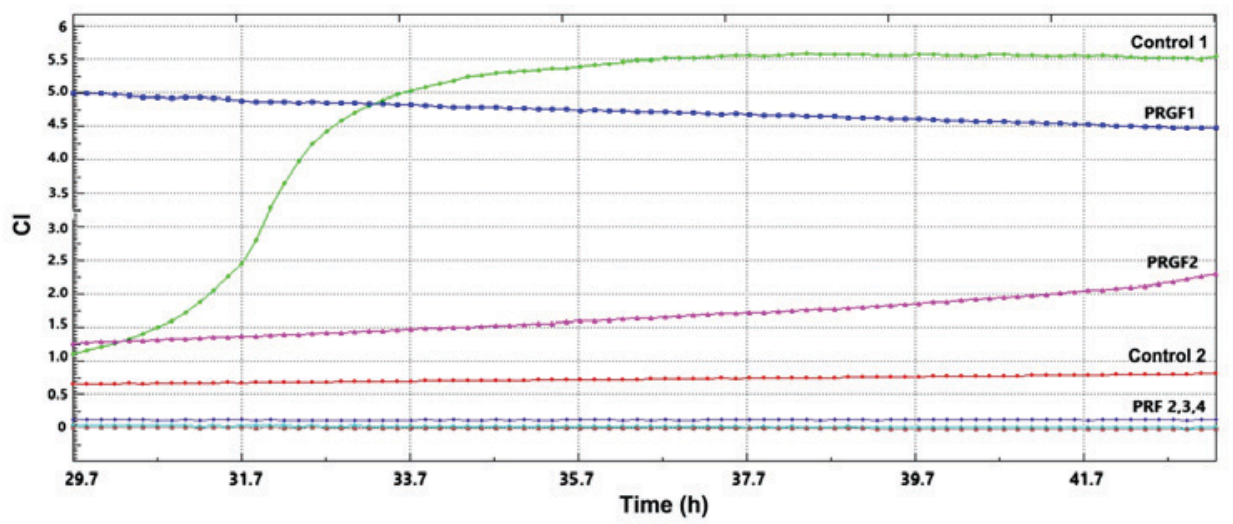

Figure 7. Fibroblast migration registered for $44 \mathrm{~h}$ in the presence of PRGF1 and 2, PRF2-4 compared to control 1 (fibroblasts migrating toward complete culture medium) and control 2 (fibroblasts migrating toward plain medium). Representation of CI mean for triplicates.

ABC dilutions. Moreover, $48 \mathrm{~h}$ cultivation in the highest dilution $(1: 48)$ of $\mathrm{ABCS}$, displayed a pattern matching one of the control cells.

The RCTA-DP system comprises the RTCA software 2.1.0 that automatically displays impedance measurements in arbitrary units and calculates automatically CI for each well. When we individually represented the obtained results (Fig. 5) some interesting findings occurred. Thus, no matter the tested dilutions, all individual ABCs have the same proliferation capacity after the first $2 \mathrm{~h}$ (Fig. 5A). Representation depicted in Fig. 5B and $\mathrm{C}$ was performed with the same $\mathrm{Y}$ axis to show the differences between CI registered at $2 \mathrm{~h}$ compared to further cultivation.

This effect becomes statistically different after $24 \mathrm{~h}$ of cultivation (Fig. 5B; p <0.001) and different activation potential was registered between individual ABCs. There is a clear dose-dependent proliferation capacity induced by ABCs, with 1:48 dilution having the lowest capacity for all the tested compounds. After $48 \mathrm{~h}$ of cultivation we register an activated proliferation, but slightly decreased compared to the $24 \mathrm{~h}$ profile (Fig. $5 \mathrm{C} ; \mathrm{p}<0.001$ ). At $48 \mathrm{~h}$, the lowest dilution of $\mathrm{ABCs}$ matches the fibroblast proliferation induced by complete medium. The ABC particularities are reflected by the $\mathrm{ABC}$ no. 6 that, from the beginning, induces the lowest proliferative capacity in fibroblasts, while $\mathrm{ABC}$ nos. 2, 3 and 5 have similar effects. We chose to represent the same CI units on the y-axis to underline approximately 8-fold increase in the proliferation capacity of fibroblasts when using 1:4 dilution. Moreover, the registered effect has a clear dose-effect pattern decreasing with the increased dilution of the compounds. This effect is sustained by the soluble components of $\mathrm{ABC}$ as cell/cell debris were removed. An identical pattern for $\mathrm{ABC}$ testing was obtained in the migration tests of fibroblasts (data not shown).

Fibroblasts migration. The migration capacity of fibroblasts is strictly linked to the capacity of wound healing. More interesting results were obtained in the migratory tests for the gel compounds (PRGF and PRF). In our system we have noted that PRGF 1 and 2 compounds develop a good migratory capacity in the first $24 \mathrm{~h}$ of cultivation, in contrast to the tested PRFs that display no proliferative action upon in vitro fibroblasts (Fig. 6). When testing further this capacity, we observed that PRGF1 and 2 diminish in their action that is surpassed by the control 1 , namely the positive control in complete cultivation medium (Fig. 7).

When investigating the automated CI performed by the software there is clear statistically different action upon fibroblasts migration. Thus, within the first $24 \mathrm{~h}$ of migration, cells display a statistically identical effect with the positive control (PRGF1), while a 50\% migration capacity from the positive control for PRGF2 (Fig. 8A). After $48 \mathrm{~h}$ the migratory 

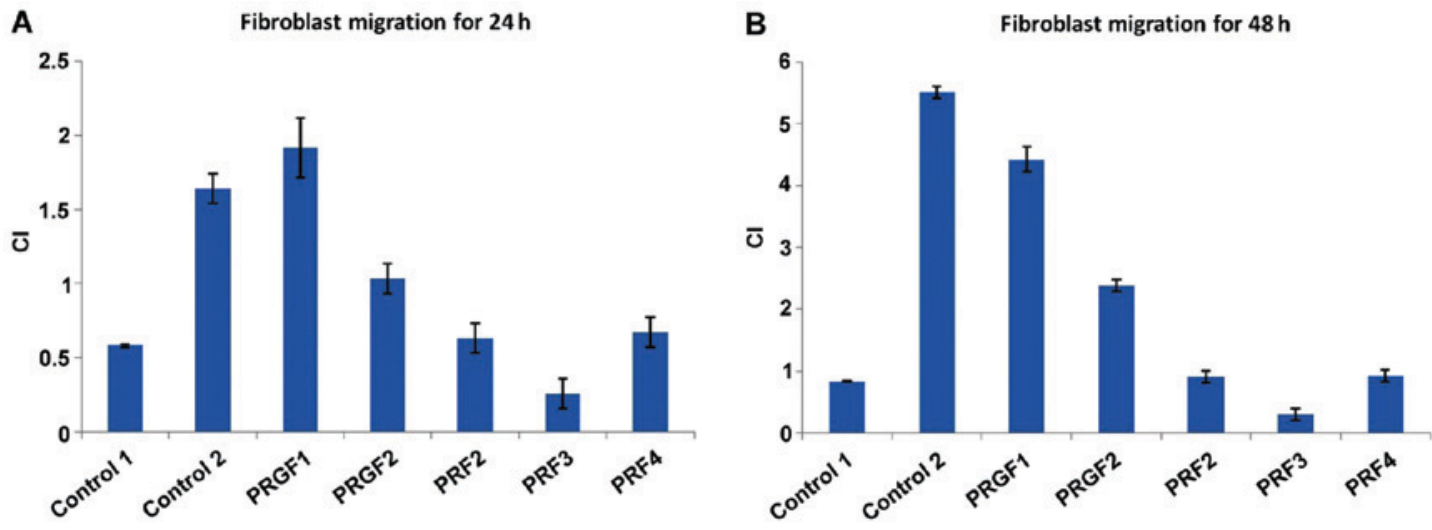

Figure 8. Cellular index (CI) calculated for cultivated fibroblasts in compounds for $24 \mathrm{~h}$ (A) and $48 \mathrm{~h}$ (B) compared to control 1 (fibroblasts migrating toward plain medium) and control 2 (fibroblasts migrating toward complete medium). CI of mean \pm SD for 3 different experiments.

capacity of PRGF1 and PRGF2 are surpassed by the positive control (Fig. 8B). Interestingly, all the tested PRFs are in the range of control 1, whether registered at 24 or $48 \mathrm{~h}$, this being the case for PRF2 and 4. PRF3 registers from the beginning an inhibition of migration capacity inflicted upon fibroblasts.

Wound healing capacity. To reinforce the migration capacity of PRGFs and PRFs we performed also a scratch test upon fibroblasts and registered their capacity to cover a $500 \mu \mathrm{m}$ gap. The highest rate of 'wound healing' was registered by the control 2, closely followed by PRGF1.

\section{Discussion}

It is known that blood components are key players in the wound healing process. Fibrin matrix binds bioactive molecules and regulates in time and space the microenvironment that will induce the healing process. As a biomimetic approach, the use of blood derivatives in regenerative strategies has awakened as a source of multiple therapeutic biomolecules. Nevertheless, and despite their clinical relevance, blood derivatives have been showing inconsistent therapeutic results due to several factors, including proper control over their delivery mechanisms. Herein, we highlight recent trends on the use biomaterials to protect, sequester and deliver these pools of biomolecules in tissue engineering and regenerative medicine approaches. Particular emphasis is given to strategies that enable to control their spatiotemporal delivery and improve the selectivity of presentation profiles of the biomolecules derived from blood derivatives rich in platelets. Finally, we discussed possible directions for biomaterials design to potentiate the aimed regenerative effects of blood derivatives and achieve efficient therapies (14). Our data confirm the knowledge that presence of the blood clot are involved in the regenerative processes; routine observations show that hematoma within the fracture site has outmost importance for callus formation and proper bone repair (15). The chemical mediators present within the blood clot, either produced by inflammatory cells captive within or by endothelial or mesenchymal cells (such as platelet-derived growth factor (PDGF), epidermal growth factor (EGF), vascular endothelial growth factor (VEGF), stromal cell-derived factor-1 (SDF-1), insulinlike growth factor-1 (IGF-1), fibroblast growth factor basic
(bFGF), transforming growth factor- $\beta$ (TGF- $\beta$ ), hepatocyte growth factor (HGF), IL-1 $\beta$, IL-6, IL-8 or monocyte chemoattractant protein 1 (MCP-1) induce fibroblasts proliferation and subsequent collagen deposition (1,16-19). We demonstrated the in vitro proliferative effect of the constituents of the blood clot on the fibroblasts in culture in a dose-dependent manner. Also we emphasize that the blood clots used in our experiments are $\mathrm{ABCs}$; there are recent studies showing that there are differences between the composition of the blood clot obtained from peripheral blood and that from bone marrow, the latter being richer in growth factors (20).

In daily practice, some clinicians used to retain $\mathrm{ABC}$ within alveolar socket after extraction; however, this procedure has no evidence-based support to date; to our knowledge, our work is the first study addressing the biological arguments in favor of this issue. PRF clots are reported in regenerative dentistry, and recently it was reported that the actual method for obtaining PRFs drives the quality and numbers of platelets within. Estimating the number of platelets entrapped in the fibrin matrix and their distribution in PRF clots and red thrombi is a measure of the thrombi quality (21). They also represent a source of growth factors such as PDGF-BB, TGF $\beta$-1, and IGF-I (22). PRGF contains important quantities of TGF- $\beta 1$, PDGF-BB, VEGF but in lower quantities than PRF while pro-inflammatory cytokines such as IL- 6 and IL-1 $\beta$ are barely present (IL-6 is present in low levels and IL-1 $\beta$ is undetectable in PRGF) $(23,24)$. The PRF compounds that were tested by us did not induce migratory action upon fibroblasts, while PRGF displayed a clear statistically significant proregenerative effect. The differences are related to the biologic composition of these growth factor sources: while both PRF and PRGF function as growth factor reservoirs due to platelets enrichment with slow release of the biomolecules, PRGF contains a very small amount (if any) of white blood cells and subsequently very few pro-inflammatory cytokines $(24,25)$. Of these cytokines with pro-inflammatory effects IL-1 $\beta$ has major role in leucocytic diapedesis in the acute phase of inflammation by stimulating expression of adhesion molecules within endothelial cells; IL-6 induces matrix degradation via matrix metalloproteinases $(26,27)$ interfere with the effect of biologically active growth factors, thus explaining the evident proliferative effect of PRGF when compared with PRP. Several other studies showed that white blood cells have 
negative effects on tissue regeneration, data supporting our findings (27-29). However, it is interesting that other authors identified significantly less evident effects of PRGF than PRF on human periosteal cells as inducers of cell proliferation in culture $(25,30,31)$.

In conclusion, cellular impedance technology reveals fibroblasts of skin origin as a perfect model to evaluate in vitro the dynamic of proliferation and migration following exposure to the tested compounds and further monitoring the efficacy in individual cases. Within this preliminary study we are highlighting the importance of investigating the cellular/ fibroblasts functionality in a network comprising both $\mathrm{ABCs}$ and platelet rich fibrin compounds in the tissue regeneration process. Moreover, studies should be expanded to other cellular models relevant for wound healing especially that oral keratinocytes could enlarge the cell population panels specific for tissue regeneration evaluation due to their interesting behavior in biomedical applications such as biotechnology of dental or orthopedic implants (32). Besides cellular models, omics tools should be additionally involved in this attempt as cell function viewed through omics lens constitute also a good barometer of tissue healing (33). Regeneration is a very complex process still insufficiently studied and both cell populations and soluble molecules should be intertwined and further connected to various additional factors, e.g., neuroendocrine factors $(34,35)$ to comprehensively decipher the tissue remodeling background.

\section{Acknowledgements}

The presented study will be integrated in the original part of $\mathrm{PhD}$ thesis of author Mihai Bucur.

\section{Funding}

This study was partially supported by a grant of Romanian Ministry of Research and Innovation, CCCDIUEFISCDI, project no. 61PCCDI/2018, code PN-III-P1-1.2PCCDI-2017-0341, within PNCDI-III.

\section{Availability of data and materials}

The datasets used and/or analyzed during the present study are available from the corresponding author on reasonable request.

\section{Authors' contributions}

$\mathrm{MB}, \mathrm{CC}, \mathrm{MN}$ and $\mathrm{SZ}$ were responsible for the research creation and design, data acquisition, analysis and interpretation of data, statistical analysis, manuscript drafting, and critical revision of the manuscript for important intellectual content. OD and CV were responsible for the data acquisition, analysis and interpretation of data, manuscript drafting, critical revision of the manuscript for important intellectual content. MC, $\mathrm{CP}$ and $\mathrm{LN}$ were responsible for analysis and interpretation of data, statistical analysis, manuscript drafting, critical revision of the manuscript for important intellectual content. EI was responsible for the research creation and design, analysis and interpretation of data, manuscript drafting, critical revision of the manuscript for important intellectual content. All authors read and approved the final manuscript.

\section{Ethics approval and consent to participate}

This study was approved by the Ethics Committee of Colentina University Hospital (no. 63/31.10.2016; Bucharest, Romania). All the enrolled subjects gave their informed consent for the present study.

\section{Patient consent for publication}

Not applicable.

\section{Competing interests}

The authors declare that they have no competing interests.

\section{References}

1. Perez RL and Roman J: Fibrin enhances the expression of IL-1 beta by human peripheral blood mononuclear cells. Implications in pulmonary inflammation. J Immunol 154: 1879-1887, 1995.

2. Butzelaar L, Niessen FB, Talhout W, Schooneman DPM, Ulrich MM, Beelen RHJ and Mink van der Molen AB: Different properties of skin of different body sites: The root of keloid formation? Wound Repair Regen 25: 758-766, 2017.

3. Greaves NS, Ashcroft KJ, Baguneid M and Bayat A: Current understanding of molecular and cellular mechanisms in fibroplasia and angiogenesis during acute wound healing. J Dermatol Sci 72: 206-217, 2013.

4. Driskell RR and Watt FM: Understanding fibroblast heterogeneity in the skin. Trends Cell Biol 25: 92-99, 2015.

5. Häkkinen L, Larjava $H$ and Koivisto L: Granulation tissue formation and remodeling. Endod Topics 24: 94-129, 2011.

6. Glim JE, van Egmond M, Niessen FB, Everts V and Beelen RH: Detrimental dermal wound healing: What can we learn from the oral mucosa? Wound Repair Regen 21: 648-660, 2013.

7. Larjava H, Wiebe C, Gallant-Behm C, Hart DA, Heino J and Häkkinen L: Exploring scarless healing of oral soft tissues. J Can Dent Assoc 77: b18, 2011.

8. Wong JW, Gallant-Behm C, Wiebe C, Mak K, Hart DA Larjava $\mathrm{H}$ and Häkkinen L: Wound healing in oral mucosa results in reduced scar formation as compared with skin: Evidence from the red Duroc pig model and humans. Wound Repair Regen 17: 717-729, 2009.

9. Mah W, Jiang G, Olver D, Cheung G, Kim B, Larjava H and Häkkinen L: Human gingival fibroblasts display a non-fibrotic phenotype distinct from skin fibroblasts in three-dimensional cultures. PLoS One 9: e90715, 2014.

10. Mah W, Jiang G, Olver D, Gallant-Behm C, Wiebe C, Hart DA, Koivisto L, Larjava H and Häkkinen L: Elevated CD26 expression by skin fibroblasts distinguishes a profibrotic phenotype involved in scar formation compared to gingival fibroblasts. Am J Pathol 187: 1717-1735, 2017.

11. Noor Mohamed R, Basha S and Al-Thomali Y: Efficacy of platelet concentrates in pulpotomy - a systematic review. Platelets 29: 440-445, 2018.

12. Neagu M, Constantin C, Tampa M, Matei C, Lupu A, Manole E, Ion RM, Fenga C and Tsatsakis AM: Toxicological and efficacy assessment of post-transition metal (Indium) phthalocyanine for photodynamic therapy in neuroblastoma. Oncotarget 7: 69718-69732, 2016.

13. Tampa M, Matei C, Caruntu C, Poteca T, Mihaila D, Paunescu C, Pitigoi G, Georgescu SR, Constantin C and Neagu M: Cellular impedance measurement - novel method for in vitro investigation of drug efficacy. Farmacia 64: 430-434, 2016.

14. Beyeler J, Schnyder I, Katsaros C and Chiquet M: Accelerated wound closure in vitro by fibroblasts from a subgroup of cleft lip/palate patients: Role of transforming growth factor- $\alpha$. PLoS One 9: e111752, 2014.

15. Mendes BB, Gómez-Florit M, Babo PS, Domingues RM, Reis RL and Gomes ME: Blood derivatives awaken in regenerative medicine strategies to modulate wound healing. Adv Drug Deliv Rev 129: 376-393, 2018 
16. Ghiasi MS, Chen J, Vaziri A, Rodriguez EK and Nazarian A Bone fracture healing in mechanobiological modeling: A review of principles and methods. Bone Rep 6: 87-100, 2017.

17. Campbell RA, Vieira-de-Abreu A, Rowley JW, Franks ZG, Manne BK, Rondina MT, Kraiss LW, Majersik JJ,Zimmerman GA and Weyrich AS: Clots are potent triggers of inflammatory cell gene expression: Indications for timely fibrinolysis. Arterioscler Thromb Vasc Biol 37: 1819-1827, 2017.

18. Distler O, Pap T, Kowal-Bielecka O, Meyringer R, Guiducci S, Landthaler M, Schölmerich J, Michel BA, Gay RE, Matucci-Cerinic M, et al: Overexpression of monocyte chemoattractant protein 1 in systemic sclerosis: Role of platelet-derived growth factor and effects on monocyte chemotaxis and collagen synthesis. Arthritis Rheum 44: 2665-2678, 2001.

19. Lee ME, Rhee KJ and Nham SU: Fragment E derived from both fibrin and fibrinogen stimulates interleukin-6 production in rat peritoneal macrophages. Mol Cells 9: 7-13, 1999.

20. Szaba FM and Smiley ST: Roles for thrombin and fibrin(ogen) in cytokine/chemokine production and macrophage adhesion in vivo. Blood 99: 1053-1059, 2002.

21. Tezono K, Sarker KP, Kikuchi H, Nasu M, Kitajima I and Maruyama I: Bioactivity of the vascular endothelial growth factor trapped in fibrin clots: Production of IL-6 and IL-8 in monocytes by fibrin clots. Haemostasis 31: 71-79, 2001.

22. Shoji T, Nakasa T, Yoshizuka M, Yamasaki T, Yasunaga Y, Adachi $\mathrm{N}$ and Ochi M: Comparison of fibrin clots derived from peripheral blood and bone marrow. Connect Tissue Res 58: 208-214, 2017.

23. Kitamura $\mathrm{Y}$, Watanabe T, Nakamura M, Isobe $\mathrm{K}$, Kawabata $\mathrm{H}$, Uematsu K, Okuda K, Nakata K, Tanaka T and Kawase T: Platelet counts in insoluble platelet-rich fibrin clots: A direct method for accurate determination. Front Bioeng Biotechnol 6: 4, 2018.

24. Dohan DM, Choukroun J, Diss A, Dohan SL, Dohan AJ, Mouhyi J and Gogly B: Platelet-rich fibrin (PRF): a second-generation platelet concentrate. Part II: platelet-related biologic features. Oral Surg Oral Med Oral Pathol Oral Radiol Endod 101: e45-e50, 2006.

25. Masuki H, Okudera T, Watanebe T, Suzuki M, Nishiyama K, Okudera H, Nakata K, Uematsu K, Su CY and Kawase T: Growth factor and pro-inflammatory cytokine contents in platelet-rich plasma (PRP), plasma rich in growth factors (PRGF), advanced platelet-rich fibrin (A-PRF), and concentrated growth factors (CGF). Int J Implant Dent 2: 19, 2016.

26. Nishiyama K, Okudera T, Watanabe T, Isobe K, Suzuki M, Masuki H, Okudera H, Uematsu K, Nakata K and Kawase T: Basic characteristics of plasma rich in growth factors (PRGF): Blood cell components and biological effects. Clin Exp Dent Res 2: 96-103, 2016.
27. Anitua E, Zalduendo M, Troya M, Padilla S and Orive G: Leukocyte inclusion within a platelet rich plasma-derived fibrin scaffold stimulates a more pro-inflammatory environment and alters fibrin properties. PLoS One 10: e0121713, 2015.

28. Contassot E, Beer HD and French LE: Interleukin-1, inflammasomes, autoinflammation and the skin. Swiss Med Wkly 142: w13590, 2012.

29. Sundararaj KP, Samuvel DJ, Li Y, Sanders JJ, Lopes-Virella MF and Huang Y: Interleukin-6 released from fibroblasts is essential for up-regulation of matrix metalloproteinase-1 expression by U937 macrophages in coculture: Cross-talking between fibroblasts and U937 macrophages exposed to high glucose. J Biol Chem 284: 13714-13724, 2009.

30. Abnave P and Ghigo E: Role of the immune system in regeneration and its dynamic interplay with adult stem cells. Semin Cell Dev Biol: Apr 9, 2018 (Epub ahead of print).

31. Nguyen HX, Hooshmand MJ, Saiwai H, Maddox J, Salehi A, Lakatos A, Nishi RA, Salazar D, Uchida N and Anderson AJ: Systemic neutrophil depletion modulates the migration and fate of transplanted human neural stem cells to rescue functional repair. J Neurosci 37: 9269-9287, 2017.

32. Calenic B, Greabu M, Caruntu C, Nicolescu MI, Moraru L, Surdu-Bob CC, Badulescu M, Anghel A, Logofatu C and Boda D: Oral keratinocyte stem cells behavior on diamond like carbon films. Rom Biotechnol Lett 21: 11914-11922, 2016.

33. Boda D: Cellomics as integrative omics for cancer. Curr Proteomics 10: 237-245, 2013.

34. Lupu M, Caruntu A, Caruntu C, Papagheorghe LML, Ilie MA, Voiculescu V, Boda D, Constantin C, Tanase C, Sifaki M, et al: Neuroendocrine factors: The missing link in non-melanoma skin cancer (Review). Oncol Rep 38: 1327-1340, 2017.

35. Caruntu C, Boda D, Constantin C, Caruntu A and Neagu M: Catecholamines increase in vitro proliferation of murine B16F10 melanoma cells. Acta Endocrinol (Buc) 10: 545-558, 2014.

This work is licensed under a Creative Commons Attribution-NonCommercial-NoDerivatives 4.0 International (CC BY-NC-ND 4.0) License. 